\title{
Fundamental perspectives on development of conductive blends based on quaternized polysulfone: Optical and dielectric response ${ }^{\dagger}$
}

\author{
Anca Filimon ${ }^{1,}{ }^{*}$, Adina Maria Dobos ${ }^{1}$ and Valentina Elena Musteata ${ }^{1}$ \\ 1 "Petru Poni" Institute of Macromolecular Chemistry, 41A Grigore Ghica Voda Alley, 700487 Iasi, Romania; \\ capataanca@yahoo.com \\ * Correspondence: capataanca@yahoo.com; Tel.: +40-232-217-454 \\ + Presented at the The 21st International Electronic Conference on Synthetic Organic Chemistry, 1-30 \\ November 2017.
}

Academic Editor: name

Received: date; Accepted: date; Published: date

\begin{abstract}
New blends based on ionic polysulfones for advanced applications, derived from quaternized polysulfone and cellulose acetate phthalate, were prepared. The optical and dielectric response was investigated according to chemical and microstructural aspects. All studied films develop two relaxation processes, i.e., $\gamma$ and $\beta$ relaxation, involving different enthalpy and entropy contributions induced by their chemical structures. Moreover, the electrical conductivity of the studied blends can be explained in terms of band conduction mechanisms through band gap representation. The outcomes highlight the electrical performances and importance of new polysulfone blends uses as good candidates for ionic exchange membranes in industrial applications.
\end{abstract}

Keywords: quaternized polysulfones; conductive blends; optical properties; dielectric response; conductivity; ionic exchange membranes

\section{Introduction}

A novel generation of organic materials with electrical and optical properties similar to those of the metals and inorganic semiconductors but which exhibit attractive characteristics associated with those of the conventional polymers, e.g., easy synthesis and flexible processing, promises to become the conductive polymers.

The conductive polymers represent dynamic structures with complex properties which have gained a great interest in various scientific fields because of their vast versatility. On the other hand, they possess good electrical and optical properties allowing an excellent control of the electrical stimulus. Furthermore, a great advantage of these types of polymers is that the physicochemical properties can be tailored to the specific necessities required by different applications, such as materials for electrostatic charge dissipation [1], ionic exchange membrane, fuel cells [2,3], and embedded capacitors [4].

Considering the excellent properties [5,6] and implicitly, the possibilities of use in a wide variety of applications from biomedicine to electronics, namely membranes for hemodialysis, ionic exchange, or gas separation, medical accessories (i.e., surgical trays, nebulizers, humidifiers), food processing equipment, and/or used in the electrical and electronics components, for example as a dielectric in capacitors [7,8], polysulfones (PSFs) are recommended as proper conductive materials. Nevertheless, there are also drawbacks in the use of these polymers in some applications, the main disadvantage being their relative hydrophobic character. Thus, the synthesis routes, by PSFs 
modification, especially the chloromethylation $[9,10]$ and quaternization reactions $[11,12]$, and specific design techniques through the introduction of another polymer in the blend [6] allow a compromise between the hydrophobicity and hydrophilicity, leading to materials with improved properties suitable for targeted applications. Additionally, to enhance the membranes properties, as well as for better electrical, thermal and mechanical characteristics, polymer blend is an efficient concept. From this perspective, the direct blending of the functionalized polysulfone containing quaternary ammonium groups (PSFQ) with cellulose acetate phthalate (CAP) has been used as strategy to develop new conductive blends based on polysulfone with predictable and controlled properties for applications as ionic exchange membranes.

It is assumed that the quaternization effect and choosing of an appropriate additive significantly improve the ionic conductivity and also could optimize the dielectric properties required by the ionic exchange membrane. Therefore, ionic polysulfone (PSFQ) obtained by the quaternization reaction of chloromethylated polysulfone (CMPSF) with tertiary amine, $\mathrm{N}, \mathrm{N}$-dimethylbutylamine (DMBA), leads to significant improvements besides the hydrophilicity, flexibility, biocompatibility, and tensile strength, the transparence features and electrical conductivity. Additionally, CAP choosing as hydrophilic and pore-forming additive represents an important tool in the workability and membrane performance.

Although the blends based on polysulfones were previous investigated for membrane applications [13], in literature no report concerning the effects induced by the structural and compositional characteristics of the polymer blends on the optical and dielectric properties is available. In order to elucidate these aspects, optic measurements and dielectric spectroscopy analysis, are used in the study of the quaternized polysulfone/cellulose acetate phthalate blend. Therefore, the main objective of this research is to evaluate some key parameters, such as the absorption coefficient and dielectric constant of films from analyzed blend on a wide frequency range, influenced not only by the structure and nature of the polymers, but also by the composition and preparation methods. Moreover, the study realized on PSFQ/CAP blend provides a new insight on analysis and understanding of the polarization and conductivity mechanisms by designing desired conditions of transparency, improved electron interactions, and conductivity proper for better electrical performances required by the ionic exchange membranes.

\section{Materials and Methods}

\subsection{Materials}

Aromatic polysulfone (PSF) supplied by Union Carbide Company, Texas (UDEL-3500) was used in the synthesis of chloromethylated polysulfones (CMPSF) and subsequently, of ionic polysulfones containing quaternary ammonium side groups (PSFQ) [14,15]. The detailed procedure of the polycondensation reactions was presented in previous study [12]. The characteristics of the synthesized polysulfones are presented in Table 1.

Table 1. Characteristics ${ }^{1}$ of the polysulfone and synthetized polysulfones.

\begin{tabular}{ccccccc}
\hline Sample/Properties & DS & $\mathbf{C l} \mathbf{( \% )}$ & $\mathbf{C l}_{\mathbf{i}} \mathbf{( \% )}$ & $\mathbf{N} \mathbf{( \% )}$ & $\mathbf{m}_{\mathbf{0}}$ & $\overline{\mathbf{M}}_{\mathbf{n}}(\mathbf{g} / \mathbf{m o l})$ \\
\hline PSF & - & - & - & - & 443 & 39,000 \\
CMPSF & 1.03 & 7.42 & - & - & 492 & 29,000 \\
PSFQ & - & - & 5.44 & 2.48 & 582 & 28,000 \\
\hline
\end{tabular}

${ }^{1}$ Characteristics include substitution degree, DS, chlorine, ionic chlorine, and nitrogen contents, molecular weights of structural units, $m_{0}$, and number-average molecular weights, $\bar{M}_{n}$, of polysulfone and functionalized polysulfones.

The ionic chlorine and nitrogen contents, formed after the reaction of CMPSF with $\mathrm{N}$, Ndimethylbutylamine, were determined analytically. The total chlorine content was determined by Schöninger modified method [16] and ionic chlorine content by potentiometric titration (Titrator TTT1C Copenhagen) with $\mathrm{AgNO}_{3}$ aqueous solutions $0.02 \mathrm{~N}$. The quaternization reaction of CMPSF 
occurs at a transformation degree close to $98 \%$, thus, considering thus, that almost all chloromethylenic groups were quaternized.

Cellulose acetate phthalate (CAP, high purity $\geq 99.5 \%$ ) was purchased from Sigma-Aldrich, USA, and used as a hydrophilic and pore-forming additive in order to improve the membranes properties. This polymer has number-average molecular weight, $\overline{\mathrm{M}}_{\mathrm{n}}=2,534 \mathrm{~g} / \mathrm{mol}$, and a degree of substitution for acetyl and phthaloyl groups of 1.07 and 0.77 , respectively. N-methyl-2-pyrrolidone (NMP) (stated purity of $99.5 \%$, Sigma-Aldrich) was used as solvent.

The general chemical structures of the polymers used in this work are illustrated in Scheme 1.

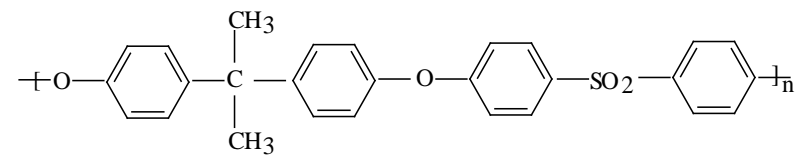

PSF

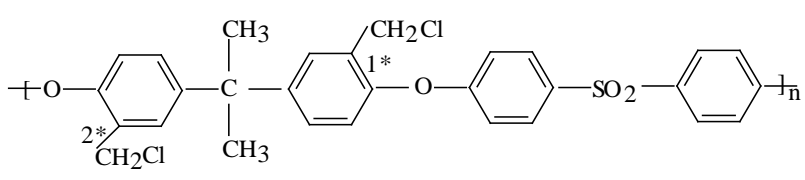

CMPSF

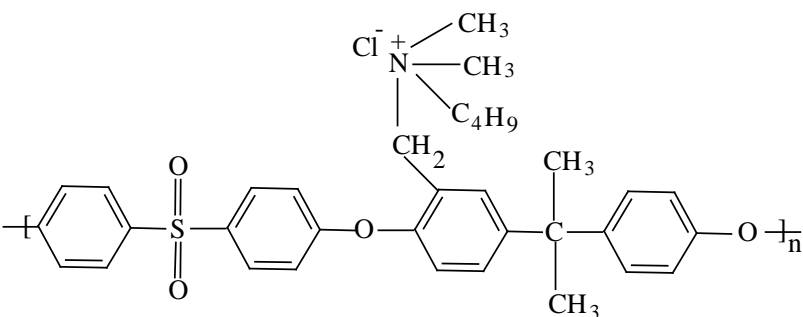

PSFQ

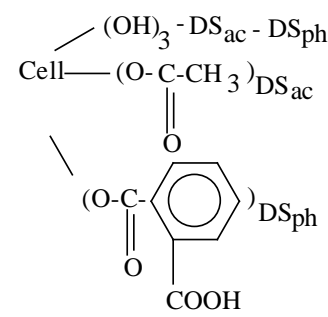

CAP

Scheme 1. Chemical structures of monomer units of the polysulfone (PSF), synthetized polysulfones (CMPSF - chloromethylated and PSFQ - quaternized polysulfone), and CAP - cellulose acetate phthalate. The chloromethylation reaction of PSF may occur in position $1^{*}$, when DS $\leq 1$ or $2^{*}$, when DS $>1$.

\subsection{Films Preparation}

The films used in the study, with a thickness of around $50 \mu \mathrm{m}$, were prepared by solution-casting technique. Homogeneous solutions of PSFQ and CAP, with known concentration of $20 \mathrm{~g} / \mathrm{dL}$, were prepared in NMP under continuous stirring for $2 \mathrm{~h}$. PSFQ/CAP blends were achieved by mixing the two solutions of PSFQ and CAP in different ratios and subsequently cast on a glass plate and solidified, initially by slow drying in saturated atmosphere of the used solvents, and finally under vacuum for 2 days at $50^{\circ} \mathrm{C}$. The 70/30 wt./wt. composition of PSFQ/CAP blend was chosen as a consequence of the structural peculiarity of polymers in the blend, as well as type of interactions, evidencing the orientation or mobility of chain segments in solution.

\subsection{Fourier Transform Infrared Spectroscopy}

Fourier transform infrared (FTIR) spectra of films were obtained using the attenuated total refraction (ATR) method in a thin $\mathrm{KBr}$ disk. The analysis was performed on a Thermo-Nicolet-6700 spectrometer (Thermo Electron Corporation), and the ATR spectra were recorded over the $500-4000$ $1 / \mathrm{cm}$ range. 


\subsection{Atomic Force Microscopy}

Atomic force microscopy (AFM) images were obtained using a Scanning Probe Microscope Solver PRO-M under tapping mode, in air, with a commercially available NSG03 rectangular shaped silicon probe (NTMDT, Zelenograd, Russia). Different scan sizes were analyzed, but the morphological features were easily observed for $2 \times 2 \mu \mathrm{m}^{2}$ scan area.

\subsection{Optical and Dielectric Measurements}

Transmittance of the studied blends based on quaternized polysulfone here under study was recorded at 200-1100 nm wavelengths, using a SPECORD 200 Analitik-Jena spectrophotometer.

Dielectric spectroscopy measurements over the 10-106 Hz frequency range were carried out using a Novocontrol Concept 40 broadband dielectric spectrometer. Temperature was controlled with a $0.1{ }^{\circ} \mathrm{C}$ device by the Novocontrol Quatro Cryosystem, in dry nitrogen atmosphere. The samples were sandwiched between two gold-coated brass electrodes and tested. The sample had around $2 \mathrm{~mm}$ thickness, with slightly larger diameters than those of the upper electrode $(20 \mathrm{~mm})$. The dielectric measurements (dielectric constant, $\varepsilon^{\prime}$, and dielectric loss, $\varepsilon^{\prime \prime}$ ) and conductivity of the PSFQ/CAP polymer blend films were performed by sweeping the frequency between 10 and $10^{6} \mathrm{~Hz}$, at fixed temperatures, at $4{ }^{\circ} \mathrm{C}$ intervals, over a temperature domain between -120 and $+120{ }^{\circ} \mathrm{C}$, at an increasing temperature rate of $2{ }^{\circ} \mathrm{C} / \mathrm{min}$. To prevent the water adsorption the dielectric spectra measurements were performed in nitrogen atmosphere.

\section{Results and Discussion}

\subsection{Microstructural Characterization of Films}

Knowledge of chemistry and structure of PSFQ and CAP films is essential for establishing and understanding of the formation process of from PSFQ/CAP blends and also, of changes induced by pure components in composite materials through the specific interactions that generate in the system. Therefore, Figure 1 shows the ATR-FTIR spectrum which proves the structures of the analyzed polymers, visualized by peaks corresponding to the characteristic groups. Thus, the infrared spectrum for PSFQ shows the characteristic absorption peaks around 2990-2940 and 2894-2850 $1 / \mathrm{cm}$ assigned to the $-\mathrm{CH}_{3}$ and $-\mathrm{CH}_{2}$ vibrations of the aliphatic units, respectively. The aromatic structure is confirmed by appearance of the absorption bands at approximately 1590 and $14801 / \mathrm{cm}$; two characteristic absorption peaks for $-\mathrm{SO}_{2}$ asymmetric stretching appear at 1300 and $12401 / \mathrm{cm}$, as a pair of split peaks, and strong absorption band around $11501 / \mathrm{cm}$ being attributed to $-\mathrm{SO}_{2}$ symmetric stretching. Moreover, the absorption band around $16701 / \mathrm{cm}$ confirms the occurrence of quaternization reaction of CMPSF with tertiary amine, the substitution of the chlorine atom by tertiary nitrogen, leading to the obtaining of quaternary nitrogen [13]. FTIR spectrum for CAP reveals typical absorption bands around 2980 and $28831 / \mathrm{cm}$ attributed to the asymmetric and symmetric stretching vibrations of methyl $-\mathrm{C}-\mathrm{H}$ groups. The adsorption peaks at about 1750, 1725, and $17011 / \mathrm{cm}$ are associated with the $-\mathrm{C}=\mathrm{O}$ carboxyl group vibrations, whereas the $-\mathrm{C}=\mathrm{C}-$ linkage of the aromatic ring showed a peak at about $15991 / \mathrm{cm}$. The characteristic bands at 1492 and 1284 $1 / \mathrm{cm}$ correspond to stretching vibrations of the $-\mathrm{C}-\mathrm{H}$ bond from the methylene groups and -C-O-Cbond of the ester groups, respectively. The -C-O- stretching vibrations of the cyclic ether structure is confirmed by appearance of the absorption bands at approximately 1140 to $1071 \mathrm{1} / \mathrm{cm}$; the absorption bands characteristic for the monosubstituted aromatic ring appearing at $7461 / \mathrm{cm} \mathrm{[17].}$ 


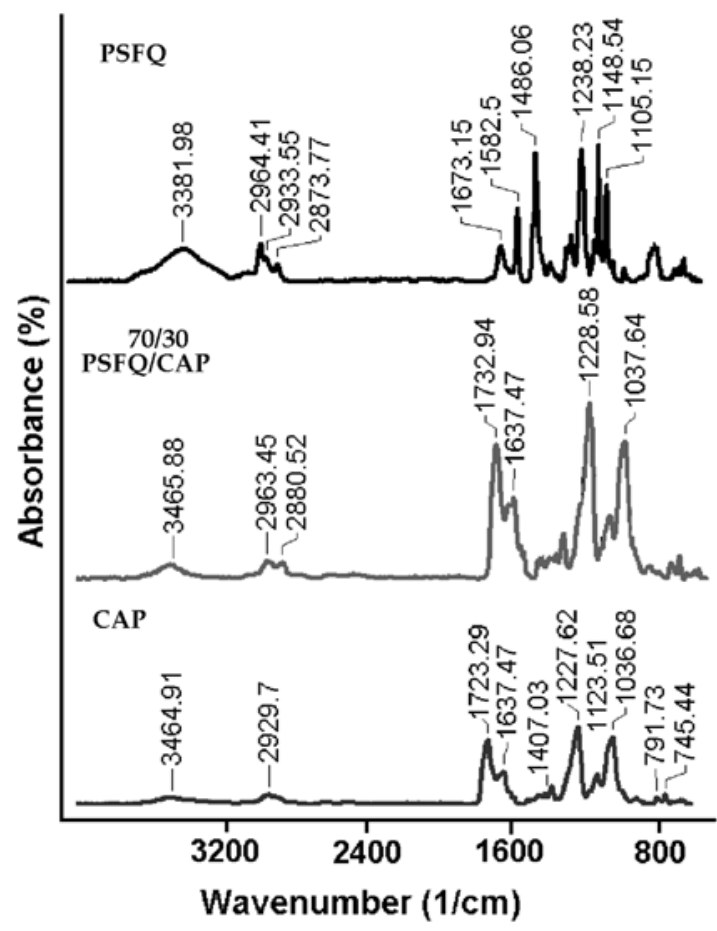

Figure 1. ATR-FTIR spectra recorded over the 4000 - $5001 / \mathrm{cm}$ range for the PSFQ, CAP and their blend at 70/30 wt./wt. mixing ratio.

Generally, a wide absorption band, generated by the valence vibrations of the $-\mathrm{OH}$ groups from the alcohol molecules associated through hydrogen bonds, can be observed in the $3100-35001 / \mathrm{cm}$ range. For 70/30 wt./wt. composition of PSFQ/CAP blend can be seen that, the -OH absorption band around of $34601 / \mathrm{cm}$ due to the inter- and intramolecular hydrogen bonding [6], moves easily to higher wavenumbers, as a result of PSFQ increase in compositions. However, a slight change of the shape, intensity, and displacements in the direction of the higher wavenumbers occurs, indicating that the addition of CAP at PSFQ solutions, in order to improve optical and electrical performances of the blends, generates new type of interactions.

Consequently, a major role in the orientation of the dipoles under the electric field action has also the properties of the films resulted from the rearrangements of the molecular chains during their drying. This rearrangement trend is confirmed by results obtained through AFM technique (Figure 2), concerning comparative analysis of surface state of the PSFQ, CAP, and PSFQ/CAP films.
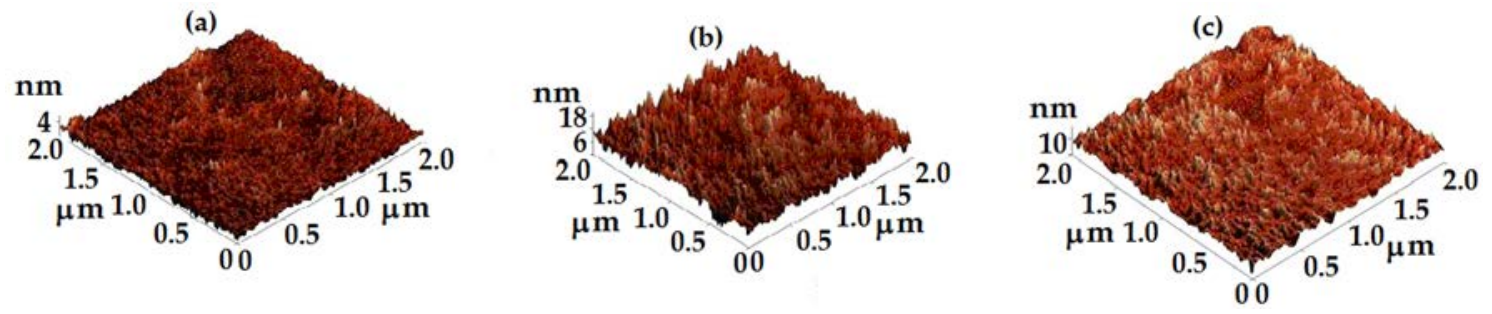

Figure 2. 3D AFM topography images of the pristine samples PSFQ (a), CAP (b), and their blend at $70 / 30 \mathrm{wt}$./wt. mixing ratio for $2 \times 2 \mu \mathrm{m}^{2}$ scan area.

3D AFM images of the studied films surface, prepared from solutions in NMP cast on glass slide, reveal porous morphology with various pore sizes ranging between $2160 \pm 280 \mathrm{~nm}$ for PSFQ > $100 \pm 17 \mathrm{~nm}$ for PSFQ/CAP $>63 \pm 12 \mathrm{~nm}$ for CAP. Since the occurrence of these pores on the PSFQ surface alternate with large flat areas, the surface area ratio, Sdr, was relatively small, namely $0.15 \%$. Instead, the CAP sample presents a well-balanced surface relief with a porous structure aspect, 
inducing a larger root mean square roughness, Sq, and Sdr. Thus, increasing of the CAP content leads to an increase of the root mean square roughness and the surface became more complex, fact shown also by the increase of the surface area ratio value. Therefore, the changing trend in films morphology can be associated with the nature of each polymeric compound from the system, in function with its dielectric response under electric field [18].

\subsection{Evaluation of the Absorption Limits of PSFQ/CAP Films: Transmission Spectra}

The materials transparency represents an important parameter in obtaining of the optical performances required by specific applications. Typical transmission spectra of the investigated polymers films (PSFQ and CAP) and 70/30 wt./wt. of PSFQ/CAP blend, were recorded over 200-1200 nm range of wavelengths, according to Figure 3. One can observe that, the transmission spectra, initiated in the ultraviolet domain, present a high transparency value of about $90 \%$.

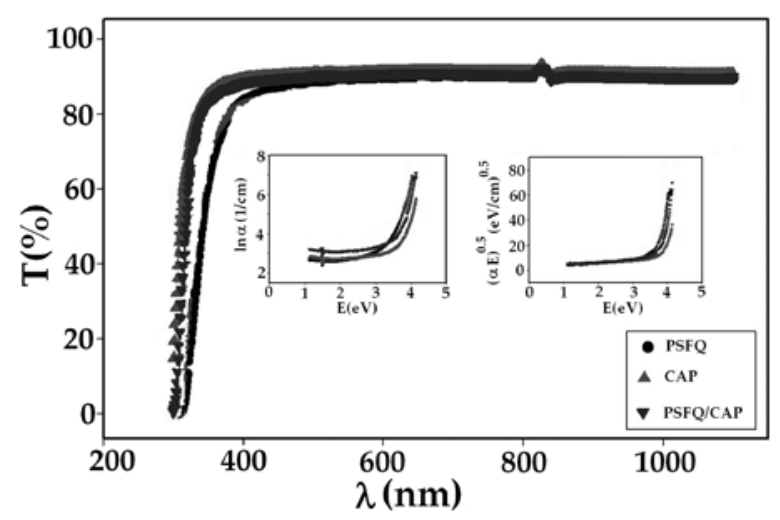

Figure 3. Typical overall transmission spectra of studied polymer films obtained from NMP solutions. Small plots represent the absorption coefficient $(\alpha)$ and Tauc dependence $(\alpha \mathrm{E})^{0.5}$ respectively, versus photon energy (E) for the same films.

Literature indicates that, for a typical amorphous semiconductor, three domains are evident in the variation of the absorption coefficient versus photon energy [19]. In the first region, the absorption coefficient, due to the inter-band transition near the band gap, describes the optical gap energy Egap. In the second region, the absorption at photon energy below to the optical gap depends exponentially on the photon energy and defines the Urbach edge energy, Eurbach, according to the idea that the sharp absorption edge is enlarged by the electric fields produced by charged impurities. The third region describes the optical absorption generated by defects appearing to energy lower than the one corresponding to the optical gap; it is sensitive to the structural properties of the materials, being defined as the Urbach tail, Etail. Etail is width of the tail of localized states (Urbach energy) corresponding to the optical transition between localized states adjacent to the valence band and extended state in the conduction band which is lying above the mobility edge. This absorption tail lies below to the exponential part of the absorption edge (the second region), and its strength and shape were found as depend on the preparation, purity, and thermal history of the material, varying slightly only in its thickness.

Particularly, the approach typical for amorphous semiconductors has been applied to the studied polymers films (equation (1)) for obtaining the absorption coefficient, $\alpha$, from transmittance data.

$$
\alpha=(1 / \mathrm{d}) \ln (1 / \mathrm{T})
$$

where $\mathrm{d}$ is film thickness.

It has been suggested that, the Urbach's rule may be used to relate the absorption coefficient to the incident photon energy [19]. Thus, from the obtained data, for the studied films the absorption coefficient is plotted as a function on photon energy, according to equation (2): 


$$
\alpha=\alpha_{0} \exp (\mathrm{E} / \mathrm{A})
$$

where $\alpha_{0}$ is a constant, $\mathrm{E}$ is photon energy.

The shape of obtained curves is similar to the behavior proposed by Tauc for typical amorphous semiconductors $[20,21]$, although the absorption level is lower than the one of the amorphous, inorganic thin films. These results are in agreement with literature data, according to which a lower absorption in the polymeric materials is due to a lower degree of bonding delocalization [22,23]. An absorption edge is a sharp discontinuity in the absorption spectrum when photon energy corresponds to the energy of an atom shell. Each of the absorption edges plotted in Figure 3 exhibit two different slopes, and a saturation region for higher energy. Thereby, parameter A becomes either Eurbach or Etail, in the high-energy region or in the low-energy region of the absorption coefficient, respectively. The absorption edges follow the Tauc power law (equation (3)), for all investigated samples, in the range over which the photon energy is higher than the optical gap energy, Egap [24].

$$
(\alpha E)^{1 / 2}=B\left(E-E_{\text {gap }}\right)^{1 / 2}
$$

where $\mathrm{B}$ is a constant.

Thus, the dependencies are used to obtain the optical gap energy for PSFQ, CAP, and 70/30 w/w PSFQ/CAP. The optical parameters values obtained for the studied samples are presented in Table 2; all parameters possess sensitive properties influenced by different factors, like the conditions of films preparation from solution, where air oxidation or the dissolving power of the solvent lead to changes in film transmittance.

Table 2. Optical parameters of the quaternized polysulfone, cellulose acetate phthalate, and $70 / 30 \mathrm{wt} . / \mathrm{wt}$. composition of their blend.

\begin{tabular}{ccccc}
\hline Sample & $\mathbf{d}(\mathbf{m m})$ & Etail $(\mathbf{m e V})$ & Eurbach $(\mathbf{m e V})$ & Egap $\left._{\mathbf{m}} \mathbf{m e V}\right)$ \\
\hline PSFQ & 0.08 & 657 & 218 & 3.78 \\
CAP & 0.06 & 741 & 231 & 3.44 \\
PSFQ/CAP & 0.04 & 599 & 240 & 3.72 \\
\hline
\end{tabular}

According to data listed in Table 2, values of Urbach energies obtained for studied polymeric films increase as the transparency of samples increase. Thus, for PSFQ film, which present less transparent, the value of Eurbach is smaller $(218 \mathrm{meV})$ comparatively with the values of the Urhach energies obtained for polymeric films with higher transparency ( $231 \mathrm{meV}$ for CAP and $240 \mathrm{meV}$ for PSFQ/CAP). At the same time, Etail energy of CAP sample presents a higher value than for PSFQ, and for their blends, being of $599 \mathrm{meV}$. Consequently, variation of these two parameters is related to the localized state induced by the atomic structures of the polymers. Possible structural defects, namely breaks, configurationally imperfections or torsions of the polymer chains, seem to be responsible for the energy described by the Urbach tail energy. Moreover, larger structural disorders may cause an increase of the Eurbach energy and Etail parameter. According to those above-mentioned, the presented data indicate that, the values obtained for Eurbach and Etail are specific for films prepared from transparent polymer solutions. Additionally, one can observe that, for the PSFQ/CAP blend, the value of Egap and the electric field produced by charged impurities, expressed by the Urbach edge energy, are especially due to the PSFQ amount, while the defect states expressed by the Urbach tail energy take close values for all samples. Overall, these results can be attributed to the structural peculiarities of the studied polymers.

\subsection{Dielectric Constant and Dielectric Loss of PSFQ/CAP Blend Films}

The dielectric properties of the blends based on quaternized polysulfones, such as the 
dielectric constant, $\varepsilon^{\prime}$, and dielectric loss, $\varepsilon^{\prime \prime}$, were measured over a wide temperature and frequency ranges. Figure 4 illustrates the dielectric behavior of PSFQ, CAP, and their blend (70/30 wt./wt. composition) in the $-120^{\circ} \mathrm{C} \div+120^{\circ} \mathrm{C}$ temperature range at different frequency $\left(10-10^{6} \mathrm{~Hz}\right)$.
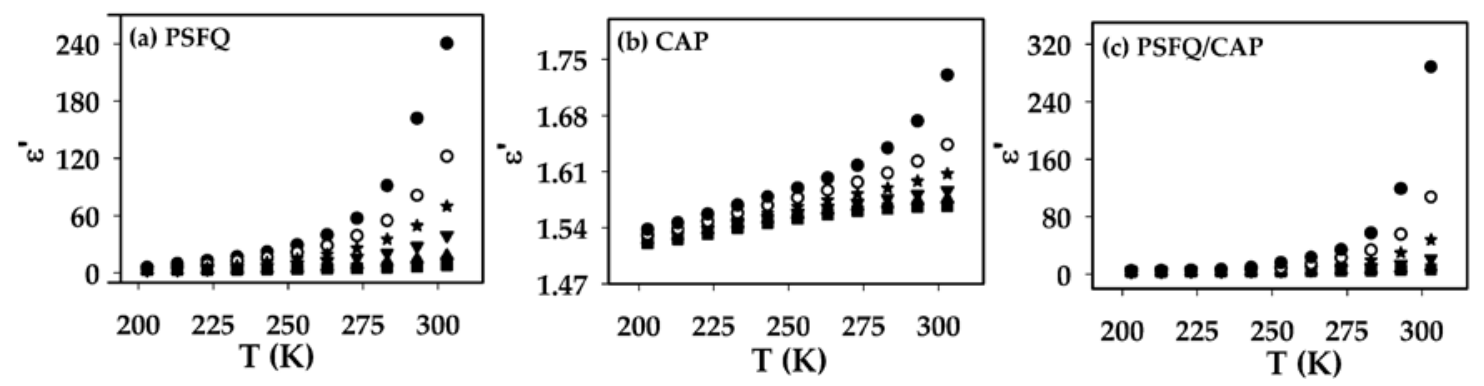

Figure 4. Variation of dielectric constant $\left(\varepsilon^{\prime}\right)$ versus temperature at different frequency (f, Hz): (匹) $10^{6}$, ( $\left.\mathbf{\Delta}\right) 10^{5}$, ( $\left.\boldsymbol{\nabla}\right) 10^{4},\left(^{*}\right) 10^{3}$, (०) $10^{2}$, (•) 10, for: (a) PSFQ, (b) CAP, and 70/30 wt./wt. composition of PSFQ/CAP blend.

According to Figure 4, the dielectric constants increase with temperature, as result of the improved polarization and more intense oscillation of the molecules present in films, arising from the dipole orientation and trapped charge carriers. In the same time, a decrease of the dielectric constants with increasing of frequency is due to the dielectric dispersion, as result of the molecules lagging behind the alternation of the electric field, at higher frequency [25]. Moreover, rapid decrease of $\varepsilon^{\prime}$ at high temperatures is generated by the increase in chaotic thermal oscillations of the molecules and to the degree of dipoles orientation. In agreement with these remarks, the $\varepsilon^{\prime}$ value for PSFQ is higher than for CAP, the electronic conjugations from the side chains of PSFQ contributing to the enhancement of $\varepsilon^{\prime}$ values. Instead, the decrease in $\varepsilon^{\prime}$ values for CAP may be attributed on the one hand, to the ability of macromolecules dipoles to orient themselves in the direction of the applied field in the low frequency range and, on the other hand, to inability of the dipoles to orient themselves in the direction of the applied field, in the high frequency range.

Over the $10-10^{6} \mathrm{~Hz}$ frequency domain, the dielectric constant values of the pure components are reflected in those of the blend. Thus, for PSFQ/CAP blend (Figure 4c), $\varepsilon^{\prime}$ takes values in 2.85-285.1 range. Consequently, one can mention that the dielectric constant depends on the chemical structure of studied polymers and composition of the polymer blends, as well as on the polarization mechanisms, being characterized by the dipole moment per unit volume induced by the electromagnetic field. Moreover, the competition between the contributions the main chain and the pendant groups are reflected in the $\varepsilon^{\prime}$ values.

Additionally, relaxation processes in blends based on quaternized polysulfone have been studied using dielectric spectroscopy in wide ranges of temperatures and frequencies. Thus, Figure 5 plots the tridimensional variation of dielectric loss, $\varepsilon^{\prime \prime}$, with frequency and temperature, for the studied polymers and their blend, where two types of relaxation, $\gamma$ and $\beta$, appear. As literature mentions, so-called secondary $\beta$ relaxation, appears at higher temperatures than $\alpha$ relaxation, being caused by the micro-Brownian motion of the main chain segments, due to the flexibility of their molecules [8]; this process imply localized and intermolecular motions. Instead, $\gamma$ relaxation, named secondary or local relaxation, appears at negative temperatures, being attributed to the molecular units motions, lower than those for $\beta$ relaxation. 

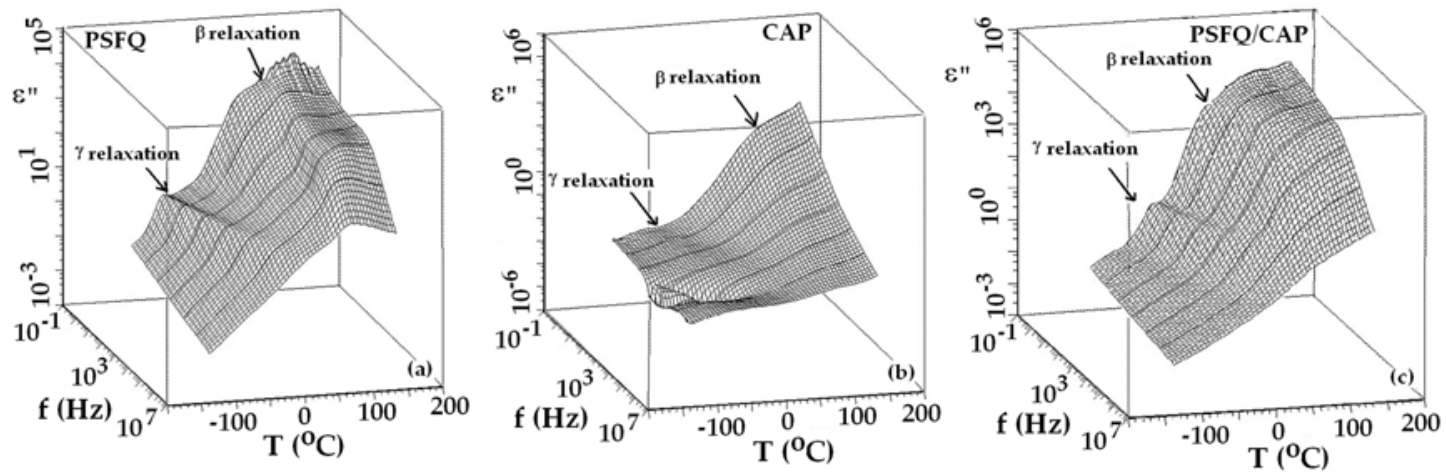

Figure 5. Dependence of dielectric loss $\left(\varepsilon^{\prime \prime}\right)$ on frequency and temperature for: (a) PSFQ, (b) PVA and (c) 70/30 wt./wt. composition of PSFQ/CAP blend.

According to Figure 5a, $\gamma$ relaxation for PSFQ is observed at lower temperatures (i.e., -80 $\div-30{ }^{\circ} \mathrm{C}$ ) in low frequency range, while $\beta$ relaxation appears at $30-100{ }^{\circ} \mathrm{C}$ and high frequencies. It can be noted the influence of the polysulfone side chain, which produces on the one hand, a decrease in the interchain attraction manifested in the starting chloromethylated polysulfone and, on the other hand, confers stiffness to the skeleton. Moreover, Figure 5b illustrates two types of relaxation for CAP; $\gamma$ relaxation at relative low temperatures $\left(-70 \div-30{ }^{\circ} \mathrm{C}\right)$ and $\beta$ relaxation in $40-115{ }^{\circ} \mathrm{C}$ temperature range. One can say that both $\beta$ and $\gamma$ relaxations of CAP related to the chain motions appear to be governed by different parameters. Thus, $\gamma$ relaxation intensity of CAP is reduced, possibly some polar groups are removed from the samples during heating. Therefore, the $\gamma$ relaxation temperature is very sensitive to changes in the chemical structure, and implicitly in microstructural characteristics, such as interchain distance, fractional free volume [26,27]. $\gamma$ and $\beta$ processes are initiated by localized motions of the side groups of atoms or molecular segments, no contribution from the backbone of the main chain being noticed. In addition, two relaxation processes occur in polymers blend (Figure 5c), as result of the relaxations temperatures at which the two processes occur in the case of pure components, caused by low-amplitude non-cooperative motion of kinetic units in side groups and a low-temperature caused by the large-scale cooperative motion of kinetic units in the main polymer chain.

\subsection{Electrical Conductivity}

The electrical conductivity modification with temperature and frequency is dependent on the structural parameters of the samples. Variation of the conductivity, $\sigma$, with frequency at different temperatures for PSFQ, CAP, and their blend is shown in Figure 6.
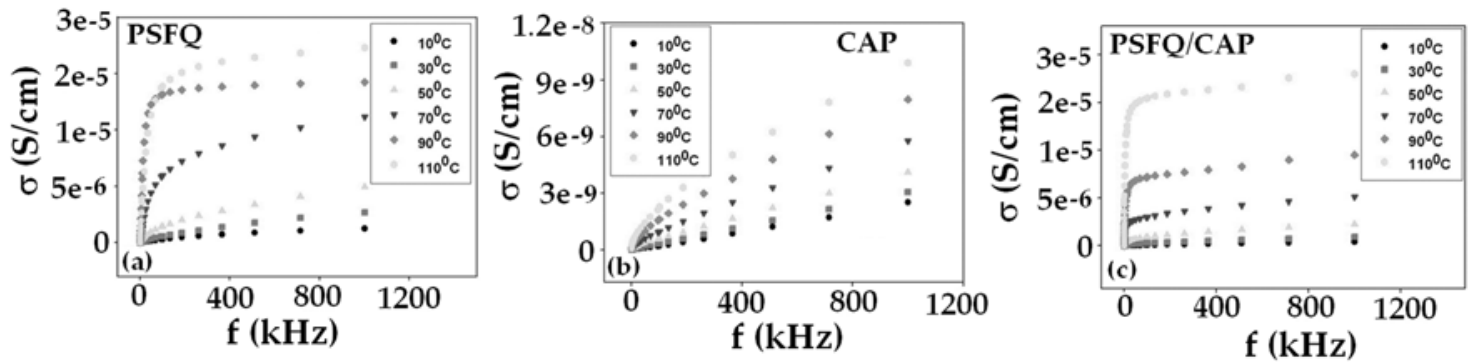

Figure 6. Dependence of conductivity $(\sigma)$ on frequency and temperature for: (a) PSFQ, (b) PVA and (c) 70/30 wt./wt. composition of PSFQ/CAP blend.

It can be noticed that, the conductivity of many materials, such as polysulfones, is described by the $\sigma \propto \mathrm{f}^{\mathrm{n}}$ dependence (the fractional exponent, $\mathrm{n}$, ranging between 0 and 1 ). According to literature data [28], the high slopes correspond to the intrinsic electrical conduction, while the 
small ones to low temperatures indicating a reduction in the impurity concentration of the samples. In the present study, $\mathrm{n}$ exponent decreases for all samples with increasing of the temperature and frequency domain, being within the $0.5<\mathrm{n}<1$ limits, at temperature close to room temperature. This conduction takes place via localized hopping of the carriers between randomly distributed trapping centers [28]. Thereby, the linear dependence of conductivity on frequency around room temperature is due to the electronic conduction via a hopping process. Deviation from linearity at high frequencies may be due to the dispersion of the charge carriers produced by dipolar relaxation.

Generally, increase in temperature leads to an increase in electrical conductivity, with low slope at high frequencies. Thus, in order to explain the influence of temperature on electrical conductivity, a model based on energy band gap representation was established. It was found that, the gap energy, $E_{g a p}=2 \mathrm{Ea}_{\text {a }}\left(\mathrm{E}_{\mathrm{a}}\right.$ is the activation energy by Arrhenius temperature dependence) take different values over the low and high temperature domain, being attributed to the hopping processes. In our investigations, the electrical conductivity, besides the electronic conduction is accompanied by an ionic conduction generated by the $\mathrm{N}, \mathrm{N}$-dimethylbutylamonium group from the quaternized polysulfone. At the same time, the thermal activation energy values of the electrical conduction $\left(E_{a}\right)$ are different from those provided by absorption spectra, being firstly a consequence of the electronic transfer mechanism appearing when changing temperature and, secondly, of the absorbed photons, electrons, and holes generators.

\section{Conclusions}

The study realized on new blends of quaternized polysulfone/cellulose acetate phthalate provides a perspective for future approaches in industrial applications, due to the dielectric properties, conductivity, and implicitly electron interactions which represent fundamental features to enhance their electrical performance. The obtained results have shown that, the dielectric and optical response-chemical structure relationship can provide information on the electronic and ionic conduction mechanisms and the molecular processes, involving different relaxations. Consequently, the findings of this study demonstrate that the blends based on quaternized polysulfones may offer important advantages for membrane applications, e.g., ionic exchange membranes.

Acknowledgments: We thank to Dr. Ecaterina Avram (1950-2016), Department of Functionalized Polymers, "Petru Poni" Institute of Macromolecular Chemistry, Iasi, Romania, for the polysulfones synthesis.

Author Contributions: Anca Filimon and Adina Maria Dobos have designed the experiments (characterization of the synthesized polysulfones, surface chemistry analysis), discussed the data, and wrote the paper. Valentina Elena Musteata has performed the dielectric spectroscopy measurements.

Conflicts of Interest: The authors declare no conflict of interest.

\section{References}

1. Kwon, J.; Kim, K.H. Comparison of the properties of waterborne polyurethane/multiwalled carbon nanotube and acid-treated multiwalled carbon nanotube composites prepared by in situ polymerization. J Polym Sci A Polym Chem 2005, 391, 3973-3985, DOI: 10.1002/pola.20897.

2. Liao, S.H.; Hung, C.H.; Ma, C.C.M.; Yen, C.Y.; Lin, Y.F.; Weng, C.C. Preparation and properties of carbon nanotube-reinforced vinyl ester/nanocomposite bipolar plates for polymer electrolyte membrane fuel cells. J Power Sources 2008, 176, 175-182, DOI: 10.1016/j.jpowsour.2007.10.064.

3. Wang, G.; Weng, Y.; Chu, D.; Chen, R.; Xie, D. Developing a polysulfone-based alkaline anion exchange membrane for improved ionic conductivity. J Membr Sci 2009, 332, 63-68, DOI: 10.1016/j.memsci.2009.01.038.

4. Pang, H.; Chen, T.; Zhang, G.; Zeng, B.; Li, Z.M. An electrically conducting polymer/graphene composite with a very low percolation threshold. Mater Lett 2010, 64, 2226-2229, DOI: 10.1016/j.matlet.2010.07.001. 
5. Barikani, M.; Mehdipour-Ataei, S. Synthesis, characterization, and thermal properties of novel arylene sulfone ether polyimides and polyamides. J Polym Sci A Polym Chem 2000, 38, 1487-1492, DOI: 10.1002/(SICI)1099-0518(20000501)38:9<1487::AID-POLA11>3.0.CO;2-U.

6. Filimon, A.; Avram, E.; Stoica, I. Rheological and morphological characteristics of multicomponent polysulfone/poly(vinyl alcohol) systems. Polym Int 2014, 63, 1856-1868, DOI: 10.1002/pi.4716.

7. Cha, D.I.; Kim, K.W.; Chu, G.H.; Kim, H.Y.; Lee, K.H.; Bhattarai, N. Mechanical behaviors and characterization of electrospun polysulfone/polyurethane blend nonwovens. Macromol Res 2006, 14, 331-337, DOI: 10.1007/BF03219090.

8. Saxena, P.; Gaur, M.S.; Shukla, P.; Khare, P.K. Relaxation investigation in polysulfone: thermally stimulated discharge current and dielectric spectroscopy. J Electrostat 2008, 66, 584-588, DOI: 10.1016/j.elstat.2008.07.002.

9. Higuchi, A.; Sugiyama, K.; Yoon, B.O.; Sakurai, M.; Hara, M.; Sumita, M.; Sugawara, S.; Shirai, T. Serum protein adsorption and platelet adhesion on pluronic $^{\mathrm{TM}}$-adsorbed polysulfone membranes. Biomaterials 2003, 24, 3235-3245, DOI: 10.1016/S0142-9612(03)00186-8.

10. Ioan, S.; Filimon, A.; Avram, E. Influence of the degree of substitution on the solution properties of chloromethylated polysulfone. J Appl Polym Sci 2006, 101, 524-531, DOI 10.1002/app.23340.

11. Ydens, I.; Moins, S.; Degee, P.; Dubois, P. Solution properties of well-defined 2-(dimethylamino)ethyl methacrylate-based (co)polymers: a viscometric approach. Eur Polym J 2005, 41, 1502-1509, DOI: 10.1016/j.eurpolymj.2005.02.002.

12. Filimon, A.; Dobos, A.M.; Avram, E.; Ioan, S. Ionic polymers based on quaternized polysulfones: hydrodynamic properties of polymer mixtures in solution. Pure Appl Chem 2014, 86, 1871-1882, DOI: 10.1515/pac-2014-0603.

13. Filimon, A.; Albu, R.M.; Stoica, I.; Avram, E. Blends based on ionic polysulfones with improved conformational and microstructural characteristics: Perspectives for biomedical applications. Composites Part B 2016, 93, 1-11, DOI: 10.1016/j.compositesb.2016.02.062.

14. Avram, E.; Butuc, E.; Luca, C.J.; Druta, I. Polymers with pendant functional group. III. Polysulfones containing viologen group. J Macromol Sci Part A Pure App Chem 1997, 34, 1701-1714, DOI: 10.1080/10601329708010036.

15. Luca, C.; Avram, E.; Petrariu, I. Quaternary ammonium polyelectrolytes. V. Amination studies of chloromethylated polystyrene with N,N-dimethylalkylamines. J Macromol Sci Part A: Chem 1988, 25, 345-361, doi: 10.1080/00222338808053373.

16. Haslan, J.; Hamilton, J.B.; Squirell, D.C.M. The determination of chlorine by the oxygen flask combustion method: A single unit for electrical ignition by remote control and potentiometric titration. Analyst 1960, 85, 556-561, DOI: 10.1039/AN9608500556.

17. Menjoge, A.R.; Kulkarni, M.G. Mechanistic investigations of phase behavior in Eudragit ${ }^{\mathrm{E}}$ blends. Int J Pharm 2007, 343, 106-121, DOI: 10.1016/j.ijpharm.2007.05.033.

18. Lv, F.; Liu, L.; Zhang, Y.; Li, P. Effect of polymer structure on the morphologies and dielectric properties of nanoporous polyimide films. J Appl Polym Sci 2015, 132, 41480, DOI: 10.1002/app.41480.

19. Ghobadi, N.; Band gap determination using absorption spectrum fitting procedure. Int Nano Lett 2013, 3, 1-4, DOI: 10.1186/2228-5326-3-2.

20. Tauc, J.; Menth, A.; Wood, D.L. Optical and magnetic investigations of the localized states in semiconducting glasses. Phys Rev Lett 1970, 25, 749-752, DOI: 10.1103/PhysRevLett.25.749.

21. Tauc, J.; Menth, A. States in the gap. J Non Cryst Solids 1972, 8-10, 569-585, DOI: 10.1016/0022-3093(72)90194-9.

22. Jarzabek, B.; Weszka, J.; Burian, A.; Pocztowski, G. Optical properties of amorphous thin films of the Zn-P system. Thin Solid Films 1996, 279, 204-208, DOI: 10.1016/0040-6090(95)08162-3.

23. Jarzabek, B.; Schab-Balcerzak, E.; Chamenko, T.; Sek, D.; Cisowski, J.; Volozhin, A. Optical properties of new aliphatic-aromatic co-polyimides. I Non Cryst Solids 2002, 299-302, 1057-1061, DOI: 10.1016/S0022-3093(01)01130-9.

24. Davis, E.A.; Mott, N.F. Conduction in non-crystalline systems V. Conductivity, optical absorption and photoconductivity in amorphous semiconductors. Philos Mag 1970, 22, 903-922, DOI: 10.1080/14786437008221061.

25. Nada, A.M.A.; Dawy, M.; Salama, A.H. Dielectric properties and AC-conductivity of cellulose polyethylene glycol blends. Mater Chem Phys 2004, 84, 205-215, DOI: 10.1016/S0254-0584(02)00418-2. 
26. Hariharan, R.; Bhuvana, S.; Sarojadevi, M. Synthesis and characterization and properties of organo soluble polyimides, bismaleimides and polyaspartimides based on 4,4-dichloro-3,3-diamino benzophenone. High Perform Polym 2006, 18, 163-184, DOI: 10.1177/0954008306056866.

27. Monetes, H.; Mazeanu, K.; Cavaille, J.Y. Secondary mechanical relaxations in amorphous cellulose. Macromolecules 1997, 30, 6977- 6984, DOI: 10.1021/ma9611329.

28. Kulanthaisami, S.; Mangalaraj, D.; Narayandass, S.K. Conduction studies on polyvinyl alcohol films. Eur Polym J 1995, 31, 969-975, DOI: 10.1016/0014-3057(95)00059-3.

(C) 2017 by the authors. Submitted for possible open access publication under the terms and conditions of the Creative Commons Attribution (CC BY) license (http://creativecommons.org/licenses/by/4.0/). 